\title{
ARTIGO
}

\section{Comunicação científica dos docentes da Universidade Federal do Maranhão no período de 1998 a 2001}

\author{
Professors' scientific communication \\ in the Federal U niversity of Maranhão, \\ Brazil, from 1998 to 2001
}

Cláudia Maria Pinho de Abreu PECEGUEIRO ${ }^{1}$

Silvana Maria de JESUS²

RE S U M O

O objetivo é a caracterização da comunicação científica na Universidade Federal do Maranhão -UFMA, no período de 1998 a 2001. Dado que o papel da ciência é buscar aproximar o homem da verdade científica, enfatiza-se a importância da divulgação dos resultados dos estudos para validação da pesquisa. Tal divulgação ocorre através da comunicação científica, envolvendo canais (formais, informais e eletrônicos) facilitadores da tramitação da informação. Neste trabalho, apresentam-se os resultados de levantamento sobre as formas de comunicação científica utilizadas pelos docentes da UFMA, via canais formais.

Palavras-chave: comunicação científica, produção científica, UFMA.

A B S T R A C T

This paper's objective was the characterization of the scientific communication which took place during the period 1998 to 2001, in the Universidade Federal do Maranhão - UFMA. Given that the role of science is to try to bring mankind closer

\footnotetext{
1 Mestre em Ciência da Informação, UnB. Professora, Universidade Federal do Maranhão. E-mail: clpecegueiro@uol.com.br

2 Graduanda, Curso de Biblioteconomia, Universidade Federal do Maranhão. E-mail: silnana@yahoo.com.br Recebido e aceito para publicação em 8/7/2003.
} 
to the scientific truth, this work emphasizes the importance of publicizing the research results in order to validate the research. The publication process occurs through the channels of scientific communication, which may be formal, informal or electronic, and will make the spreading of information easier. This paper presents the results of the survey on the forms of scientific communication via formal channels, used by the UFMA teachers,

Key words: UFMA, scientific communication, scientific production and publication.

\section{N T R O D U Ç Ã O}

A sociedade tem percebido a força que a ciência vem exercendo no desenvolvimento das nações. Sendo dinâmica e interacionista, a ciência se desdobra num emaranhado de problemas que surgem e tendem a ser solucionados pelos pesquisadores. São esses problemas que vão dar origem à pesquisa científica. Os cientistas ou pesquisadores tentam buscar soluções para os mesmos desenvolvendo estudos diversos, isto é, partem em busca da verdade, a fim de formular novos paradigmas ou reafirmar os já existentes.

A validade dos resultados de pesquisa é testada pela comunidade científica. Através de avaliações, discussões e análises, os estudos desenvolvidos por um pesquisador, após divulgados, são aceitos ou refutados. Essa atividade praticada pela comunidade científica possibilita a comunicação entre o pesquisador e seus pares.

Ziman (1979), Meadows (1999) Muller (2000) e Targino (2000) muito discutem sobre o processo de comunicação no meio científico. Com base nessas e em outras discussões, a ciência vem sendo divulgada das mais diversas formas possíveis. Através dos canais de comunicação (formais, informais, eletrônicos) os trabalhos são divulgados e apreciados pela comunidade científica. "Para sobreviver no mercado de trabalho ou para conviver com seus pares, os indivíduos são forçados a assimilar um corpo de conhecimento que se amplia a cada segundo" (TARGINO, 2000, p.7). Aí está a importância da comunicação que permite ao pesquisador trocar informações com seus pares, através dos canais informais de comunicação científica, formando os colégios invisíveis, dos canais formais que envolvem os documentos escritos e impressos e dos canais eletrônicos que englobam, tanto a comunicação científica formal (periódicos eletrônicos, livro eletrônico, entre outros, quanto a informal (correios eletrônicos, grupos de discussões).

A respeito de comunicação científica eletrônica Kuramoto et al. (2002, p.1) comenta que:
A Internet incrementou a comu- nicação entre os pesquisa- dores, propiciando uma mídia de fácil acesso e que lhes assegura rapidez e visibilidade no inter- câmbio de informações com os seus pares. Essa agilidade fez contrastar o tempo de produção e distribuição de revistas científicas impressas com a instantaneidade das publicações eletrônicas.

Devido a rapidez oferecida pelos meios eletrônicos, torna-se mais fácil para o pesquisador comunicar seus resultados sem perder muito tempo. A troca de informações é às vezes, imediata, o que dá ao pesquisador e a seus pares condições de incrementar seu trabalho com menos tempo. A velocidade com que a ciência 
vai se modificando e traçando seu caminho, força o pesquisador a estar sempre atento às novas tendências temáticas, aos novos paradigmas.

Esta pesquisa busca identificar as formas de comunicação científica, via canais formais, utilizadas pelos docentes da Universidade Federal do Maranhão (UFMA) de 1998 a 2001; classificar a literatura produzida segundo o formato (relatórios, teses, dissertações, artigos de periódico, monografias, entre outros formatos) e segundo a autoria (individual ou coletiva).

O tema proposto tenta delinear um perfil dos Professores Pesquisadores da UFMA. Esperamos com este estudo contribuir para o conhecimento e divulgação das formas de comunicação científica utilizadas por esses pesquisadores.

\section{E TO D O LO G I A}

Para desenvolver este estudo foram realizadas quatro etapas: 1) pesquisa bibliográfica; 2) aplicação de questionários junto aos docentes pesquisadores que não fazem parte do universo da pesquisa, para a realização de um pré-teste; 3) aplicação do instrumento de coleta de dados, o questionário, destinado aos docentes que integram o universo da pesquisa; 4) análise e tabulação dos dados coletados.

O universo da pesquisa é compreendido por um Departamento de cada Centro de Ensino da UFMA. A seleção foi feita a partir de um sorteio aleatório, ficando o universo composto por: Departamento de Biblioteconomia (DEBIB), representando o Centro de Ciências Sociais, com 19 docentes; Departamento de Psicologia (DEPSI), representando o Centro de Ciências Humanas, com 14 docentes; Departamento de Educação Física (DEEFIS), representando o Centro de Ciências Biológicas e da Saúde, com 15 docentes e Departamento de Matemática (DEMAT), representando o Centro Tecnológico, com 23 docentes.
Assim, do universo de 71 professores dos 4 Departamentos representantes dos quatro Centros, obtivemos um total de 26 questionários que corresponde a $37 \%$ do universo. Consideramos que $37 \%$ é bastante representativo e se encontra no nível de confiança estabelecido. Com o universo definido, partimos então para a coleta de dados que teve início com a revisão de literatura sobre o tema que auxiliou na construção e estruturação da pesquisa.

O questionário (Anexo) utilizado na coleta de dados contém tópicos que integram 8 questões, abertas e fechadas.

\section{RES U LT A D O S}

Esta etapa da pesquisa segue a linha diretriz do instrumento questionário que arrola cinco itens relacionados à identificação do respondente, formação, atividades docentes e produção científica.

\section{Identificação}

O primeiro aspecto analisado corresponde ao sexo dos docentes. Nos DEBIB e DEPSI encontramos um número superior de docentes do sexo feminino em relação ao masculino, o que não se comprova nos DEMAT e DEEFIS. Esse fato pode estar relacionado aos preconceitos existentes em relação às áreas. Ao estudar o sistema de recompensa na ciência, as especificidades e condicionantes em algumas áreas do conhecimento, Ozozco (1998, p.155-158) assegura que a condição de gênero que caracteriza as mulheres é, de fato, utilizada como uma desigualdade sexual, ou seja, em áreas de estudo masculino esse fato é mais evidente, uma vez que muitos homens consideram a mulher incapaz de crescer profissionalmente no mesmo espaço que eles ocupam. A autora acrescenta ainda que em áreas de domínio feminino, como é o caso das Ciências Humanas e Sociais (a 
exemplo da Biblioteconomia), esta prática também é evidente.

Grande parte dos docentes que integram a amostra desse trabalho, ingressaram na UFMA na década de 90 . O destaque ficou para o DEBIB, com 9 professores admitidos nesse período (Figura 1).

Percebemos que há um grande número de professores contratados mais recentemente, 19, na década de 90 , e 4 no início dos anos 2000, em relação às décadas de 70 (1 professor) e 80 (2 professores). Tal fato pode estar relacionado à data de criação da Universidade Federal do Maranhão (TÁJRA, 1985), quando a maioria do corpo docente formado nos anos 70 , já em fase de aposentadoria (25 e 30 anos de serviço) propiciou, na década de 90 , a renovação do quadro profissional.

\section{Situação funcional}

A Resolução n. 1053/91 CD que trata da progressão vertical por titulação, determina que o professor com título de mestre, ao ingressar

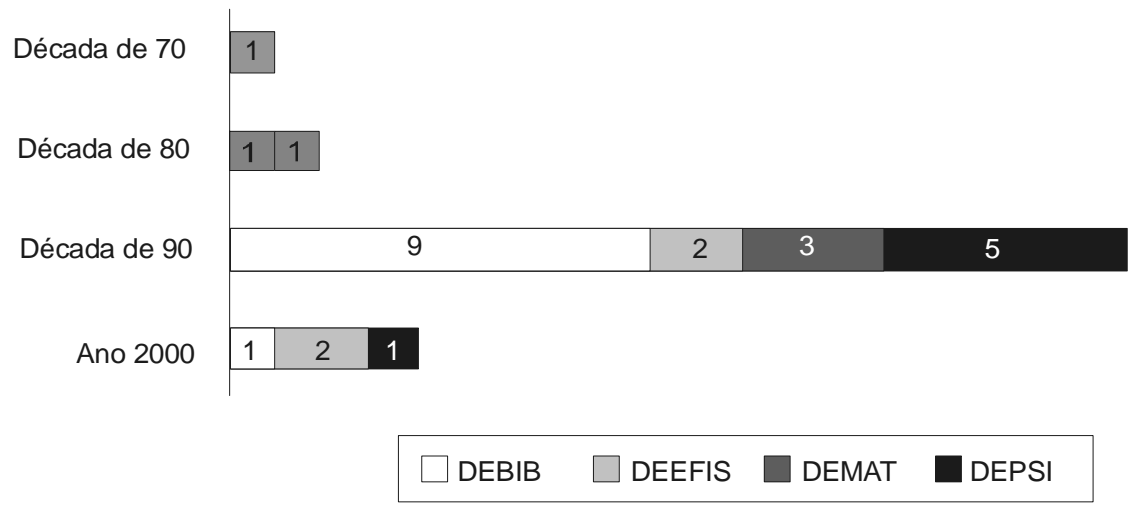

Figura 1. Distribuição dos docentes por período de admissão na UFMA.

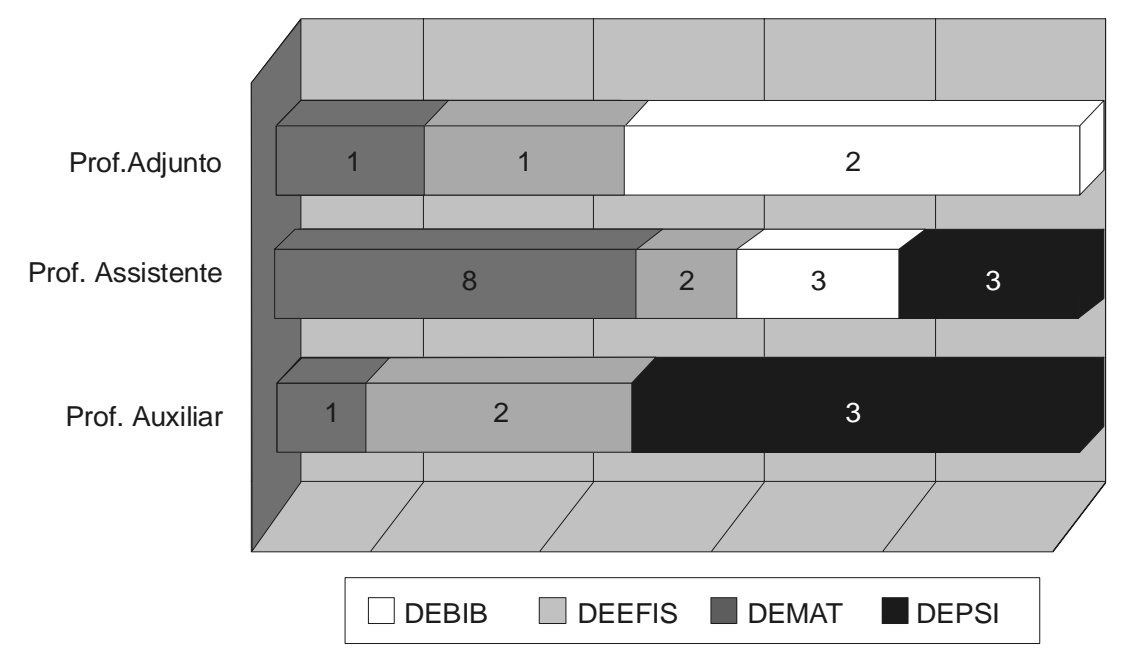

Figura 2. Distribuição dos docentes por situação funcional. 
no quadro docente da UFMA, automaticamente ingresse na categoria de Professor Assistente. O docente com título de especialista seja ingresse na categoria de Professor Auxiliar e o docente doutor, passe a integrar a categoria de Professor Adjunto. Há casos em que o tempo de serviço também influencia na categoria docente, é o caso da progressão vertical por tempo de serviço (Figura 2).

De acordo com o nosso estudo, a UFMA dispõe de um número considerável de professores titulados, haja vista que na categoria de Professor Assistente os 16 representantes têm título de mestre, e na categoria Adjunto, dos 4 representantes, 3 são doutores.

\section{Formação}

Em relação à formação acadêmica, verificamos nesta pesquisa (Figura 3), que o nível de formação dos docentes da UFMA é considerável.

\section{Atividades Docentes}

De acordo com nosso estudo, verificamos que dos 26 docentes que fazem parte da amostra, $69,23 \%$ trabalham $40 \mathrm{~h}$ semanais em regime de dedicação exclusiva, e dedicam pelo menos $20 \mathrm{~h}$ para as atividades de pesquisa. Essa quantidade diminui nos docentes que trabalham $40 \mathrm{~h}$ semanais sem regime de dedicação exclusiva, $30,77 \%$ (8), que dedicam à pesquisa, menos de $10 \mathrm{~h}$ semanais. O restante $(3,85 \%)$, não trabalha com pesquisa.

Isto demonstra que, quanto maior for 0 tempo dispensado pelo docente para o cumprimento de suas atividades acadêmicas, maior será a sua contribuição para o desenvolvimento da ciência na instituição e, conseqüentemente, poderá haver aumento na produção científica.

A quantidade de disciplinas lecionadas, tanto na graduação, quanto na pós-graduação, é muito variada, o que não nos permite fazer uma aferição detalhada. Situações como participação de professores em cursos de pós-graduação, a exemplo de Mestrado e Doutorado, fazem com que as horas em sala de aula sejam reduzidas por um determinado período de tempo.

De acordo com esta pesquisa, percebemos que os 26 docentes integrantes da amostra dedicam horas diferenciadas às atividades administrativas, de ensino, pesquisa, e extensão (Figura 4).

As atividades de ensino e administrativas dispõem de um maior número de horas semanais de cada docente. Nelas há a ocorrência dos 26 docentes pesquisados (100\%). Nas atividades de pesquisa e extensão isso não ocorre, talvez devido às dificuldades existentes para desen-

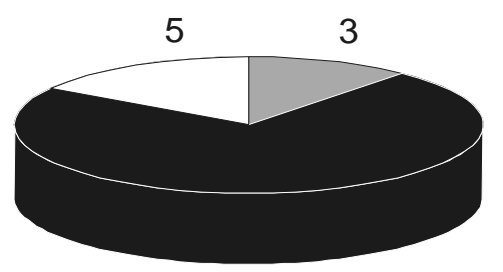

18

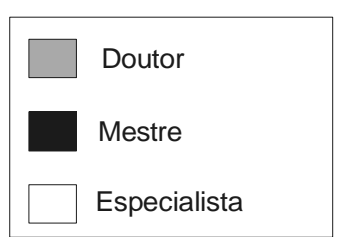

Figura 3. Qualificação dos docentes da UFMA. 
volvê-las, uma vez que requerem financiamentos internos ou externos.

\section{Produção Científica}

Produção científica é considerada por Castro (1992, p.23) como "[...] o resultado da atividade científica produzido num determinado espaço de tempo ou por pesquisador". Em uma universidade, a produção científica pode ser vista como toda a literatura resultante de pesquisa, produzida na instituição, por aqueles que trabalham em prol do seu desenvolvimento, sejam eles alunos, professores, técnico-administrativos etc.

A produção científica dos docentes da UFMA neste trabalho foi classificada de acordo com o formato (Figura 5):

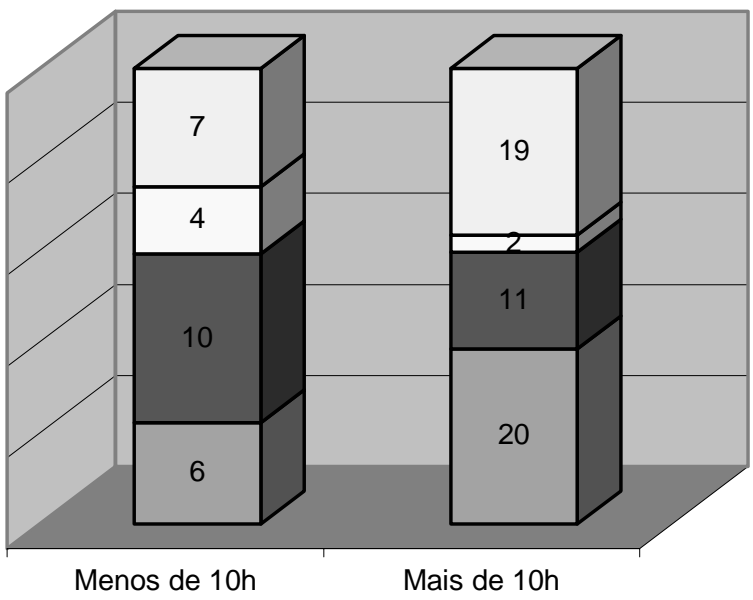

$\square$ Atividades Administrativas

\section{Extensão}

口Pesquisa

口Ensino

Figura 4. Distribuição dos docentes por atividade.

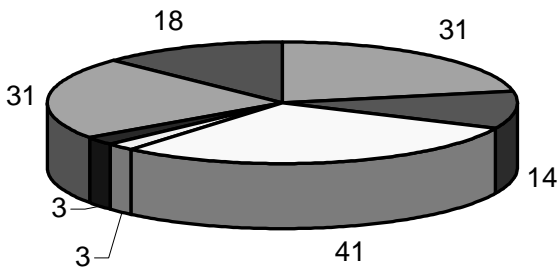

口Apostilas

口Relatórios

口Teses, dissertações e monografias $\quad$ LLivros

Capítulos de livros $\square$ Resumos em eventos

$\square$ Artigos de periódicos

Figura 5. Produtividade dos docentes da UFMA, segundo o tipo de publicação. 
a) Apostilas: materiais didáticos produzidos pelos professores para possibilitar aos alunos uma melhor compreensão das aulas ministradas por eles;

b) Relatórios técnicos: de acordo com Campello (2000, p.105), são documentos que descrevem os resultados ou andamento de pesquisas para serem submetidos à apreciação;

c) Teses, dissertações e monografias de conclusão de curso: documentos que representam os resultados de estudos oriundos de atividades de graduação e pós-graduação (CAMPELLO, 2000; ASSOCIAÇÃO BRASILEIRA..., 2001).

d) Artigos de periódicos: instrumento que possibilita uma comunicação rápida e precisa a respeito de uma experiência ou observação específica (MUELLER, 2000, p.73-74).

Dos 141 documentos produzidos nos últimos quatro anos pelos docentes da UFMA, o destaque ficou para as teses, dissertações e monografias com 41 documentos (29\%) o que vem a comprovar mais uma vez a crescente qualificação dos professores dessa instituição nesse período. Resumos em eventos e apostilas totalizam cada um 31 trabalhos (22\%). O somatório da produção científica restante totaliza 38 documentos (27\%).
Targino e Caldeira (1998), analisando a produção científica da Universidade Federal do Piauí, citam que a publicação de um documento tanto individual quanto em colaboração contribui de certo modo para percebermos as estruturas de geração de conhecimento e as formas pelas quais o documento foi organizado hierarquicamente. Eles pontuam ainda que, embora tenha havido um aumento de trabalhos publicados em colaboração, os trabalhos de autoria única ainda detêm a maior porcentagem.

Na pesquisa de Pecegueiro (2001), a respeito das temáticas dos artigos brasileiros na área da Ciência da Informação na década de 90, observamos que os artigos analisados também apresentam autoria individual mais numerosa que a múltipla.

Também constatamos nessa pesquisa que é elevado o tipo de autoria individual 101 $(71,6 \%)$ em relação a autoria coletiva, somente $40(28,4 \%)$.

Com base nos dados coletados, podemos constatar que os professores que apresentaram um nível de qualificação de especialização (5), tiveram um número menor de publicações, e os que têm mestrado (113) e doutorado (23), produziram em maior quantidade. Isso nos leva a entender que, quanto maior for o nível de

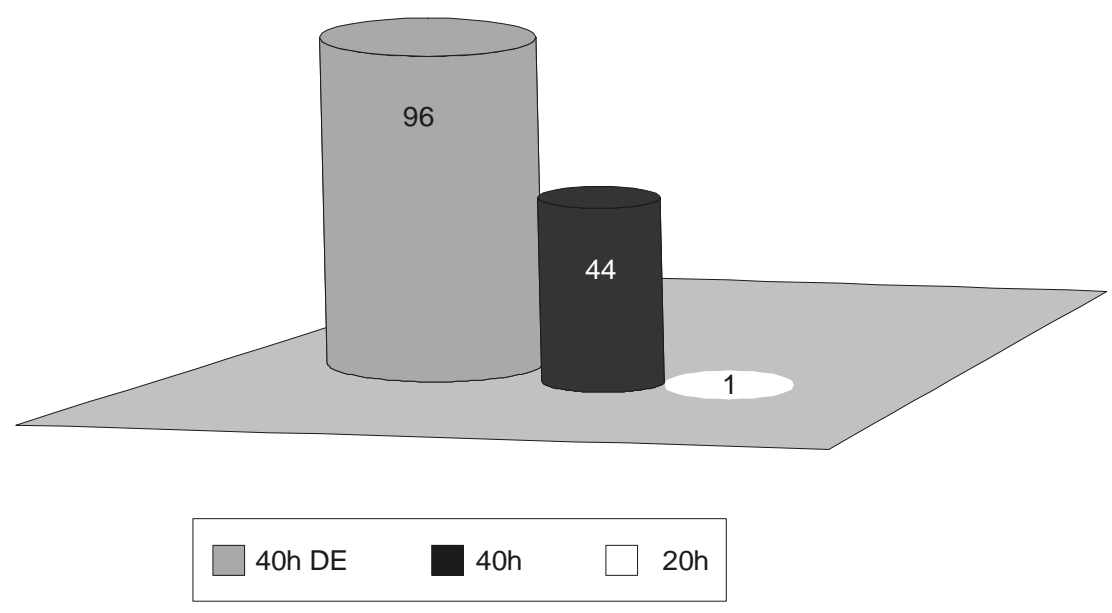

Figura 6. Produtividade dos docentes por regime de trabalho. 
formação, maior será a quantidade de publicações. Podemos comprovar esse fato nos estudos de Maciel (1982), quando afirmam que a produção científica é maior, à medida que os títulos são mais elevados.

A produtividade dos docentes está diretamente relacionada ao número de horas dedicado à UFMA. Dos 141 documentos produzidos, 96 são daqueles que trabalham $40 \mathrm{hDE}$. Os docentes de $40 \mathrm{~h}$ semanais produziram 44 trabalhos. Somente 1 trabalho foi produzido pelos professores que dedicam $20 \mathrm{~h}$ semanais à UFMA (Figura 6).

Com base nos dados da Figura 6 , podemos dizer que quanto maior for o tempo dedicado pelo docente à universidade, maior será a sua produção científica, pois a grande parte das publicações aqui apresentadas ficou com os docentes que trabalham $40 \mathrm{~h}$ semanais com dedicação exclusiva a UFMA.

\section{O N C L U SÃ O}

Com base nos resultados obtidos concluímos que:

a) ainda persistem certos preconceitos relacionados com o exercício de certas profissões por pessoas de determinado sexo;

\section{RE FER Ê N CIAS}

ASSOCIAÇÃO BRASILEIRA DE NORMAS TÉCNICAS. NBR 14724: informação e documentação: trabalhos acadêmicos: apresentação. Rio de Janeiro, 2001.

CAMPELLO, B.S. Teses e dissertações. In: CAMPELLO, B.S.; CENDÓN, B.V.; KREMER, J.M. Fontes de informação para pesquisadores e profissionais. Belo Horizonte: EDUFMG, 2000. p.121-128.

CASTRO, M.H. Produção científica dos docentes da Escola Superior de Agricultura de Lavras: análise b) a renovação do quadro de docentes da UFMA na década de 90 relaciona-se fortemente com a data de formação da universidade e aposentadoria dos professores;

c) grande número de professores Assistentes e Adjuntos é justificado pelo fato de ser elevado o número de docentes com doutorado e mestrado;

d) o regime de $40 \mathrm{~h}$ contribui para o aumento da produção científica dos docentes;

e) o tempo destinado às atividades administrativas é bastante elevado se comparado às atividades fins da universidade sendo a extensão a menos aquinhoada;

f) a produção dos docentes da UFMA atingiu uma média de 5,42 trabalhos por autor sendo que a maioria destes constitui-se sobretudo de monografias, dissertações e teses.

Os resultados obtidos não esgotaram as possibilidades a respeito da comunicação científica dos docentes da UFMA, mas mostram que a informação científica tem um valor maior do que podemos imaginar. Os levantamentos realizados, baseados em dados quantitativos, não explicam as causas dos fenômenos apresentados, limitando-se à sua proposta inicial de identificar as formas de comunicação científica via canais formais utilizados pelos docentes da UFMA no período de 1998 a 2001.

quantitativa. 1992. 169f. Dissertação (Mestrado em Biblioteconomia) - Faculdade de Biblioteconomia, Pontifícia Universidade Católica de Campinas, Campinas, 1992.

KURAMOTO, H.; CAFÉ, L.; SENA, N.K. Open archives: uma alternativa para a comunicação científica. Disponível em: <http://www.ibict.br/ arquivos abtertos/enancib.pdf>. Acesso em: 10 mar. 2002.

MACIEL, A.C. A informação científica: sua disseminação, interação informal e seus efeitos 
nos participantes de uma reunião anual de cientistas sociais brasileiros. 1982. $106 \mathrm{f}$. Dissertação (Mestrado em Ciência da Informação) - Instituto Brasileiro de Informação em Ciência e Tecnologia, Universidade Federal do Rio de Janeiro, Rio de Janeiro, 1992.

MEADOWS, A.J. A comunicação científica. Brasília: Briquet de Lemos/Livros, 1999. 268p.

MUELLER, S.P.M. A ciência, o sistema de comunicação científica e a literatura científica. In: CAMPELLO, B.S.; CENDÓN, B.V. KREMER, J.M. Fontes de informação para pesquisadores e profissionais. Belo Horizonte: EDUFMG, 2000. p.21-34.

OZOZCO, E.M. Sistema de recompensa na ciência: especificidades e condicionantes em algumas áreas do conhecimento. 1998. 210f. Tese (Doutorado em Política Científica e Tecnológica) - Pós-Graduação em Política Científica e Tecnológica, Universidade Estadual de Campinas, Campinas.

PECEGUEIRO, C.M.P.A. Temáticas dos artigos de periódicos brasileiros na área da Ciência da
Informação na década de 90. 2001. $105 f$. Dissertação (Mestrado em Ciência da Informação) - Faculdade de Estudos Sociais Aplicados, Universidade de Brasília, Brasília, 2001.

TÁJRA, L.M.C. Primeiro ciclo de estudos básicos da Universidade Federal do Maranhão: contribuições para reflexão. São Luís: EDUFMA, 1985. 108p.

TARGINO, M.G. Comunicação científica: uma revisão de seus elementos básicos. Informação \& Sociedade: estudos, João Pessoa, v.10, n.2. 2000. Disponível em: <http://www.informacaoe sociedade. ufpb.br>. Acesso em: 15 mar. 2002.

TARGINO, M.G.; CALDEIRA, P.T. Análise da produção científica em uma instituição de ensino superior: o caso da Universidade Federal do Piauí. Ciência da Informação, Brasília, v.17, n.1, p.15-25, 1998.

ZIMAN, J. O homem e a ciência: conhecimento público. Belo Horizonte: Itatiaia, 1979. 163p. Cap. 4: Educação para a ciência. 
ANEXO

\section{QUESTIONÁRIO DESTINADO AOS DOCENTES}

I- IDENTIFICAÇÃO DORESPONDENTE
1.1 Sexo:
( ) Masculino
( ) Feminino

1.2 Departamento:

1.3 Admitido pela UFMA em:

1.4 Situação funcional:
( ) Prof. Assistente
( ) Prof. Adjunto
( ) Prof. Auxiliar

II - FORMAÇÃO

2.1 Formação Acadêmica (indicar apenas a última)
( ) Graduação - Curso/Área:
Instituição:
( ) Especialização - Curso/Área:
Instituição:
( ) Especialização - Curso/Área: Instituição:
( ) Mestrado - Curso/Área: Instituição:
( ) Doutorado - Curso/Área: Instituição:

\section{III - ATIVIDADES DOCENTES}

3.1 Regime de trabalho
( ) $20 \mathrm{~h}$
( ) $40 \mathrm{~h}$
( ) 40h DE

3.2 Disciplinas lecionadas nos últimos 4 Anos
( ) Graduação. Quantas?
( ) Pós-graduação. Quantas?

3.3 De acordo com sua carga horária semanal, como é feita a distribuição do seu tempo para:

$\begin{array}{lr}\text { Ensino: } & \text { horas } \\ \text { Pesquisa: } & \text { horas } \\ \text { Extensão: } & \text { horas } \\ \text { Atividades Administrativas: } & \text { horas }\end{array}$


IV - PRODUÇÃOCIENTÍFICA

4.1 MARCAR COMUM XOS DOCUMENTOS PUBLICADOS NOS ÚLTIMOS 4 ANOS EQUANTIFICÁ-LOS CONSIDERANDO OTIPO DE AUTORIA

( ) Apostila.

Produzidas individualmente

Produzidas em colaboração

( ) Relatórios técnicos científicos.

Produzidos individualmente

Produzidos em colaboração

( ) Teses.

Produzidas individualmente

Produzidas em colaboração

) Dissertação.

Produzidas individualmente

Produzidas em colaboração

( ) Monografias.

Produzidas individualmente

Produzidas em colaboração

( ) Livros.

Produzidos individualmente

Produzidos em colaboração

( ) Capítulos de livros.

Produzidos individualmente

Produzidos em colaboração

( ) Resumos em eventos nacionais

Produzidos individualmente

Produzidos em colaboração

( ) Resumos em eventos internacionais.

Produzidos individualmente

Produzidos em colaboração

( ) Artigos em periódicos científicos.

Produzidos individualmente

Produzidos em colaboração 
